\title{
Reprogramming liver-stem WB cells into functional insulin-producing cells by persistent expression of Pdx1- and Pdx1-VP16 mediated by lentiviral vectors
}

\author{
Dong-Qi Tang ${ }^{1, *}$, Shun Lu ${ }^{1, *}$, Yu-Ping Sun ${ }^{1}$, Enda Rodrigues ${ }^{1}$, Wayne Chou ${ }^{2}$, Cheryl Yang ${ }^{1}$, \\ Li-Zhen $\mathrm{Cao}^{1}$, Lung-Ji Chang ${ }^{2}$ and Li-Jun Yang ${ }^{1}$ \\ ${ }^{1}$ Department of Pathology, Immunology and Laboratory Medicine, University of Florida College of Medicine, \\ Gainesville, FL, USA and ${ }^{2}$ Department of Molecular Genetics and Microbiology, University of Florida College \\ of Medicine, Gainesville, FL, USA
}

\begin{abstract}
Adenovirus-mediated transient expression of the pancreatic duodenal homeobox transcription factor Pdx1 in mouse liver activates pancreatic endocrine and exocrine genes, the latter reportedly resulting in severe hepatitis. Expression of a super-active form of Pdx1 or Pdx1-VP16 selectively transdifferentiates hepatic WB cells into functional pancreatic beta-like insulin-producing cells, without evidence of exocrine differentiation. No study has systematically compared the transdifferentiation efficiency of Pdx1 and Pdx1-VP16 at the cellular and molecular level. Comparisons can be ambiguous when vectors harboring a transcription factor cDNA have differing extents and duration of gene expression. In view of the remarkable capacity of lentiviral vector (LV) for delivering and integrating transgene into both dividing and nondividing cells, we transduced rat hepatic stem cell-like WB cells with LV-Pdx1 or LV-Pdx1-VP16, and then used the limiting-dilution technique to clone singlecell-derived cell lines that stably express either Pdx1 or Pdx1-VP16. With these cell lines, we studied: (a) the expression of Pdx1 or Pdx1-VP16 protein by Western blotting and immunocytochemistry; (b) the repertoire of long-term expression of Pdx1- or Pdx1-VP16-induced pancreatic gene expression using RT-PCR methods; and (c) their capacity to serve as beta-cell surrogates in restoring euglycemia in streptozotocin-treated diabetic mice. We found that cell lines expressing either Pdx1 or Pdx1-VP16 long-term exhibited similar profiles for expression of genes related to pancreatic development and beta-cell function, and reversed hyperglycemia in diabetic mice. We also examined short-term expression of Pdx1 or Pdx1-VP16, and the results demonstrated that expression of Pdx1-VP16 is more efficient in initiating liver-to-endocrine pancreas transdifferentiation. Our findings demonstrate: (a) that the LV system is highly effective in producing persistent expression of Pdx1 or Pdx1-VP16 in WB hepatic cells; and (b) long-term, persistent expression of either Pdx1 or Pdx1-VP16 is similarly effective in converting hepatic stem cells into pancreatic endocrine precursor cells that, upon transplantation into diabetic mice, become functional insulin-producing cells and restore euglycemia.
\end{abstract}

Laboratory Investigation (2006) 86, 83-93. doi:10.1038/labinvest.3700368; published online 14 November 2005

Keywords: Pdx1; Pdx1-VP16; transdifferentiation; insulin-producing cells; liver WB cells; pancreas

Pancreatic duodenum homeobox protein-1 (Pdx-1) is a homeodomain protein of 283 amino acids whose expression is absolutely required for the pancreas to develop. This protein is now recognized to be a critically important transcription factor involved in

Correspondence: Dr L-J Yang, MD, Department of Pathology, Immunology and Laboratory Medicine, University of Florida College of Medicine, 1600 SW Archer Road, PO Box 100275, Gainesville, FL 32610-0275, USA.

E-mail: yanglj@pathology.ufl.edu

* These authors contributed equally to this work.

Received 15 August 2005 revised and accepted 3 October 2005; published online 14 November 2005 regulating the expression of multiple genes essential for proper functioning of the beta cells in the endocrine pancreas (islets of Langerhans) that produce insulin. ${ }^{1}$ In humans and other animal species, the embryonic development of the pancreas requires $\mathrm{Pdx} 1$, as demonstrated by the identification of an individual with pancreatic agenesis resulting from a mutation that impaired the transcription of a functionally active $\mathrm{Pdx} 1$ protein. ${ }^{2}$ In adults, $\mathrm{Pdx} 1$ is essential for normal pancreatic islet function as suggested by its regulatory action on the expression of a number of pancreatic genes, including insulin, somatostatin, islet amyloid polypeptide, the glucose transporter type 2 and glucokinase. ${ }^{3}$ 
Recent studies show that ectopic and transient Pdx1 expression in liver in vivo using adenoviruses results in both exocrine and endocrine pancreatic gene expression, with insulin- as well as glucagonproducing cells located in the proximity of hepatic central veins. Such gene therapy persistently reduces streptozotocin (Stz)-induced hyperglycemia in diabetic mice, ${ }^{4,5}$ suggesting that reprogramming liver cells to pancreas by $\mathrm{Pdx} 1$ expression may serve as a reliable approach for generating endocrinepancreatic tissue to replace malfunctioning beta cells in patients with diabetes. However, other studies show that Pdx1 expression alone is not sufficient in converting liver cells into pancreatic insulin-producing cells (IPCs), and causes severe hepatitis in the mouse liver ${ }^{6}$ and liver dysmorphogenesis. $^{7}$ This is probably due to the lack of selectivity of Pdx1-induced liver-to-pancreas conversion. Our recently published study showed that Pdx1-VP16, a fusion protein of Pdx1 and the activating domain of the viral VP16 transcription factor, ${ }^{8}$ can selectively transdifferentiate rat hepatic stem-like WB cells into IPCs without any evidence of pancreatic exocrine differentiation. ${ }^{9}$ These studies raise an important and clinically relevant question: Do Pdx1 and Pdx1-VP16 have similar potency in their ability to mediate liver-to-pancreas transdifferentiation?

We recognized the possibility that Pdx1-VP16 may only appear to be more effective when delivered by a vector having a relatively low transfection potential. Up to now, no study has systematically compared Pdx1- and Pdx1-VP16-mediated liver cell transdifferentiation by probing the underlying changes at molecular and cellular levels. We therefore generated lentiviral vectors (LVs) containing both mouse Pdx1 and its modified form mouse Pdx1-VP16 and transduced rat hepatic stem-like WB cells with the LVs. Single-cell-derived clones containing either Pdx1 or Pdx1-VP16 genes were derived. Gene expression profiles and the ability of the Pdx1- or Pdx1-VP16-expressing cells to become IPCs were systematically compared under various in vitro culture conditions and followed by in vivo implantation in diabetic animals. Our study shows that a persistent expression of Pdx1 or Pdx1-VP16 are equally effective in reprogramming hepatic cells into pancreatic precursor cells that, upon transplantation into diabetic mice, further differentiate into mature IPCs and reverse hyperglycemia.

\section{Materials and methods}

\section{Plasmid Constructs and Lentiviral Production}

LV constructs containing mouse $P d x 1$ or rhGFP genes were constructed by inserting the entire mouse $P d x 1$ coding sequence (gift of CV Wright) or rhGFP (Stratagene La Jolla, CA, USA) into the pTYF vector cassette under control of the elongation factor- $1 \alpha(\mathrm{eEF}-1 \alpha)$ promoter. A Pdx1-VP16 expres- sion vector was constructed by fusing the coding sequence for the 80-residue VP16 activation domain to the C-terminus of mouse $\mathrm{Pdx} 1 .{ }^{10}$ Lentivirus was produced and the titer was determined as previously described. ${ }^{11-13}$

\section{Transduced Cell Lines}

Rat liver epithelial WB cells (passage 23), representing the cultured counterpart of liver stem-like cells, ${ }^{14,15}$ were derived from normal liver cells from an adult male Fisher 344 rat. WB cells were transduced with Pdx1, Pdx1-VP16, or rhGFP genes at a multiplicity of infection (MOI) of 20. The singlecell-derived clones containing either Pdx1 or Pdx1VP16 were obtained by limiting dilution. Cell transduction by LVs was conducted as previously described. ${ }^{11-13}$ Transduction efficiency was more than $95 \%$, as determined by transducing WB cells with $L V$-rhGFP at a MOI of 20 for $72 \mathrm{~h}$ by fluorescence microscopy. WB cells (passage 9) were transduced with either LV-Pdx1 or LV-Pdx1-VP16 at a MOI of 20 and the cells were used to harvest total RNA for short-term gene expression studies.

\section{Cell Culture Conditions}

WB-derived cell lines were maintained in RPMI 1640 medium supplemented with 10\% FCS and $11.1 \mathrm{mM}$ D-glucose. The single-cell-derived clones were selected by Western blotting with anti-Pdx1 or anti-VP16 antibodies. Three clones from both Pdx1 and Pdx1-VP16-positive cells were expanded and maintained, either in a low-glucose condition $(5 \mathrm{mM})$ or a high-glucose condition $(20 \mathrm{mM})$. The total RNA and cellular proteins were harvested from the cells monthly to the end of the observation (3 months). The rat INS-1 cell line (clone 832/13) (gift from Christopher B Newgard) has been maintained continuously for 1.5 years in RPMI 1640 medium and served as a positive control for rat $\beta$-cells. ${ }^{16}$

\section{Western Blotting}

The cell clones were expanded into six-well plates and the cellular proteins were obtained by collecting the cells into $2 \times$ SDS sample buffer and heating at $95^{\circ} \mathrm{C}$ for $5 \mathrm{~min}$. Pdx1 and Pdx1-VP16 fusion proteins were detected according to our previously published methods. ${ }^{9}$ In brief, cellular proteins from each clone were separated by SDS-PAGE using $12 \%$ Tris-HCl gels (Bio-Rad) and transferred to the filter paper. Proteins blotted with rabbit anti-Pdx1 serum (1:5000) (a gift of CV Wright), or anti-VP16 (1:200, BD Pharmagen), followed by HRP-conjugated secondary antibody (1:20000). All proteins were visualized by enhanced chemiluminescence (ECL) Western blotting detection kit (Amersham Bioscience). 


\section{RT-PCR}

Total RNA was prepared from cells using TRIreagent, and gene expression was determined by RT-PCR as previously described. ${ }^{9,17}$ The forward and reverse PCR primers were designed to be located in different exon(s) and have been previously described (Table 1). ${ }^{9}$

\section{Measurement of Insulin by ELISA}

Insulin content in cell lysates and insulin release into the culture medium from the cells were measured in triplicate as previously described. ${ }^{9,17}$ In brief, various WB-derived cells were cultured under low- or high-glucose conditions. The culture medium was collected for the determination of static insulin release. The cell lysates were collected in RIPA buffer and the protein concentrations were determined. Insulin release and insulin contents were determined using a commercial ELISA kit for rat insulin (Linco).

\section{Cell Transplantation}

Male NOD-scid mice (8-10 weeks old) were made hyperglycemic by i.p. injections of Stz at $50 \mu \mathrm{g} / \mathrm{g}$ body weight daily for 5 days, as previously described. ${ }^{10}$ When blood glucose levels reached
$>350 \mathrm{mg} / \mathrm{dl}$, mice were transplanted with WBPdx1, WB-Pdx1-VP16 or WB-rhGFP cells $\left(2 \times 10^{6}\right.$ cells/mouse) into the left renal capsular space. The blood glucose levels were monitored regularly at 1600 in nonfasting condition. Mice were killed around day-40 post-transplantation to assess metabolic activity and morphologic characteristics. The control hyperglycemic mice were terminated around day-40 post-transplantation. Intraperitoneal glucose tolerance test (IPGTT) was performed as previously described $^{9}$ in WB-derived cell transplanted mice after blood glucose levels were normalized as well as in normal control mice.

\section{Statistical Analysis}

The statistical significance of our experimental findings was analyzed by using an independent sample $t$-test, using a $P$-value of less than 0.05 for the data to be considered significant.

\section{Results}

\section{Generation of Pdx, or Pdx1-VP16-Positive Single-Cell-Derived Hepatic Cell Lines}

To systematically compare the effects of Pdx1 or Pdx1-VP16 on reprogramming liver-to-pancreas transdifferentiation, we first generated cell lines

Table 1 List of primer information for RT-PCR

\begin{tabular}{|c|c|c|c|c|c|c|}
\hline Genes & Forward primer & Reverse primer & $\begin{array}{c}\text { PCR size } \\
(b p)\end{array}$ & $\begin{array}{l}\text { GenBank } \\
\text { aсc. no. }\end{array}$ & $\underset{\left({ }^{\circ} \mathrm{C}\right)}{\mathrm{Tm}}$ & $\begin{array}{l}\text { No. of } \\
\text { cycles }\end{array}$ \\
\hline Actin & cgt aaa gac ctc tat gcc aa & agc cat gcc aaa tgt ctc at & 351 & V01217 & 56 & 35 \\
\hline HNF-1 & ttc taa gct gag cca gct gca gac g & gct gag gtt ctc cgg ctc ttt cag a & 275 & X54423 & 56 & 35 \\
\hline $\operatorname{mPdx} 1$ & tac aag ctc gct ggg atc act & gca gta cgg gtc ctc ttg tt & 309 & X_74342 & 56 & 35 \\
\hline rPdx1 & cgg cca cac agc tct aca agg & gag gtt acg gca caa tcc tgc & 667 & NM_022852 & 56 & 35 \\
\hline Ngn3 & ctg cgc ata gcg gac cac agc ttc & ctt cac aag aag tct gag aac acc ag & 324 & NM_021700 & 58 & 35 \\
\hline NeuroD & ctt ggc caa gaa cta cat ctg g & gga gta ggg atg cac cgg gaa & 225 & NM_019218 & 57 & 35 \\
\hline Nkx2.2 & cag cag cga caa ccc cta c & aag agc act cgg cgc ttc $\mathrm{c}$ & 204 & NM_010919 & 58 & 35 \\
\hline $\operatorname{Pax} 4$ & cag cag cat gga cca gct tgg & ctc ctg taa tgc ccg cag gac & 214 & XM_133023 & 55 & 35 \\
\hline Nkx6.1 & atg gga aga gaa aac aca cca gac & taa tcg tcg tcg tcc tcc tcg ttc & 280 & AF357883 & 58 & 35 \\
\hline Pax6 & gag aca gat tac tct ccg agg & acc aca cct gta tcc ttg ctt ag g & 465 & NM_013001 & 55 & 35 \\
\hline Isl-1 & cgg gag gat ggg ctt ttc tg & agc tgc ttt tgg ttg agc aca g & 191 & NM_017339 & 56 & 35 \\
\hline MafA & gac atc tcc cca tac gaa gtg & ccg cta cta cgt ttc tta tct & 462 & NM_008814 & 55 & 35 \\
\hline Glut-2 & tcc agt aca ttg cgg act tcc & ggt gta gtc cta cac tca tg & 304 & J03145 & 58 & 35 \\
\hline GK & aag gga aca aca tcg tag ga & cta tgg cgg tct tca tag ta & 126 & X53589 & 56 & 35 \\
\hline Insulin I & tac aat cat aga cca tca gca & cag ttg gta gag gga gca gat & 355 & $\mathrm{Gi}: 204956$ & 56 & 35 \\
\hline Insulin II & agc cct aag tga cca gct aca & tgc caa ggt ctg aag gtc ac & 343 & V01243 & 56 & 35 \\
\hline $\mathrm{PP}$ & gtc gca tac tac tgc ctc tcc & aga cag aag gga ggc tac aaa tcc & 336 & NM_012626 & 57 & 35 \\
\hline Glucagon & gac cgt tta cat cgt ggc gg & cgg ttc ctc ttg gtg ttc atc aac & 249 & NM_012707 & 58 & 35 \\
\hline Somatostatin & atg ctg tcc tgc cgt ctc $\mathrm{C}$ & tcg agt tgg cag acc tct g & 277 & NM_012659 & 56 & 35 \\
\hline GLP-1R & tct ctt ctg caa ccg aac ct & ctg gtg cag tgc aag tgt ct & 351 & S75952 & 58 & 35 \\
\hline SUR-1 & aag atc atg cac ttg tct act & aga cag cag gaa cag cgg tgt & 593 & AF039595 & 56 & 35 \\
\hline SNAP-25 & agt agt ggc cag cca gcc tg & atc tgg cga ttc tgg gtg tca & 200 & NM_030991 & 57 & 35 \\
\hline Kir6.2 & acc acg ctg gtg gac ctc aag & gca cca cct gca tat gaa tgg & 481 & RNŪ44897 & 60 & 35 \\
\hline IAPP & ggc tgt agt tcc tga agc tt & aag gtt gtt gct gga gcg aа & 260 & NM_012586 & 56 & 35 \\
\hline ChromA & act aag gtg atg aag tgt gt & tct cta cag tgt cct tgg ag & 353 & NM_021655 & 56 & 35 \\
\hline ALB & gcc cta ccc aca aag cct cag & gtg gct ttc tgt tgc tgt tca & 540 & NM_172320.1 & 56 & 35 \\
\hline CK19 & ggg gag ctg gtg gtt aag atc & gct cag tca caa gtt cca cct & 469 & AY $\overline{46} 4140$ & 58 & 35 \\
\hline Hexokinase & tga acc acg aga aga acc aga & aca atg tta gca tca tag tcc & 322 & NM_012734.1 & 56 & 35 \\
\hline $\mathrm{TGF} \alpha$ & aca gct cgc tct gct agc gct & gga tct tca gac cac tgt ctc & 469 & NM_012671 & 56 & 35 \\
\hline
\end{tabular}


a Pdx1-transduced single-cell derived clones

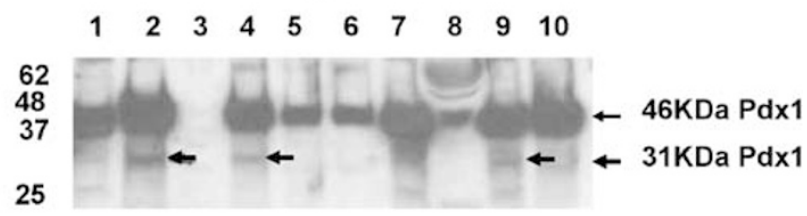

b Pdx1-VP16-transduced single-cell derived clones

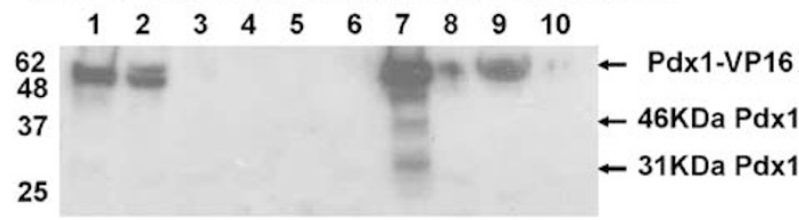

Figure 1 Generation of single-cell-derived Pdx1 or Pdx1-VP16expressing cell clones. Rat hepatic WB cells were transduced by lentiviral Pdx1- or Pdx1-VP16-vector and single-cell-derived positive clones for either Pdx1 or Pdx1-VP16 were isolated by limiting dilution. The expression of Pdx1 or Pdx1-VP16 was confirmed by separating cellular proteins via SDS-PAGE, transferring to the filter membrane, and blotting with anti-Pdx1 polyclonal antibody (1:5000). (a) Pdx1-tranduced single-cellderived clones. (b) Pdx1-VP16-transduced single-cell-derived clones. Arrows indicate the positions for active Pdx1 (46 kDa), inactive Pdx1 $(31 \mathrm{kDa})$, and a fusion protein of Pdx1-VP16 $(\sim 52 \mathrm{kDa})$.

derived from stably transfected single cells. Briefly, rat hepatic WB cells (passage 23) were grown to 70$80 \%$ confluence and transduced with LV containing Pdx1, Pdx1-VP16, or rhGFP that were constructed, produced, concentrated, and titered as described previously. ${ }^{11-13}$ At 3 days after transduction, the cells were seeded into 96-well plates by a limiting dilution to obtain single-cell clones. A total of 10 single-cell clones from either Pdx1 or Pdx1-VP16transduced cells were randomly selected, expanded, and characterized for their expression of Pdx1 or Pdx1-VP16 fusion protein by Western blotting with anti-Pdx1 or anti-VP16 antibodies. As shown in Figure 1a, nine of $10 \mathrm{Pdx} 1$-transduced WB cell clones expressed the appropriate $\mathrm{Pdx} 1$ proteins. Five out of 10 selected Pdx1-VP16-transduced clones expressed Pdx1-VP16 fusion protein, as confirmed by both anti-Pdx1 and anti-VP16 antibodies (Figure 1b). Pdx1-positive clones express a predominantly active form of Pdx1 (46 kDa), and the inactive 31-kDa Pdx1 form was expressed in clones 2, 4, and 9 (Figure 1a). Similarly, Pdx1-VP16positive clones predominantly express Pdx1-Vp16 fusion protein (upper arrow), and clone 7 also expressed both forms of endogenous $\mathrm{Pdx} 1$ as indicated by lower arrows (Figure $1 \mathrm{~b}$ ).

\section{Persistent Expression of Pdx1 or Pdx1-VP16 Proteins in Single-Cell-Derived Clones}

To determine whether the expression of Pdx1 or Pdx1-VP16 in the isolated cell clones is persistent, three clones were selected from Pdx1+ or Pdx1VP16 + cell lines. These cells were continuously expanded for 3 months (15-18 passages). The cell lysates were collected by the end of 3 month's culture. The expression of Pdx1 or Pdx1-VP16 fusion protein was determined. Figure 2a shows a persistent Pdx1 or Pdx1-VP16 protein expression after 3-month culture in these hepatic WB-derived cell lines, as revealed using Western blot by antiPdx1 antibody. To determine the nuclear localization of Pdx1 or Pdx1-VP16, the cells were spun down onto slides and immunocytochemistry was performed using anti-Pdx1 antibody. Figure 2b shows dark-brown nuclear staining of Pdx1 protein present in both Pdx1- and Pdx1-VP16-expressing cells. A similar pattern is also seen in positive control INS-1 cells but not in WB-GFP cells, indicating that these exogenous $\mathrm{Pdx} 1$ or Pdx1VP16 proteins are able to translocate to the nucleus to exert their transcription function.

\section{Gene Expression Profiles of Short-Term Expression of Pdx1 and Pdx1-VP16}

To determine the molecular difference in the gene expression profiles in both short-term and long-term Pdx1- or Pdx1-VP16-expressing cells, we first transduced WB cells with LV-Pdx1 or LV-Pdx1VP16 at a MOI of 20. Total RNA from the freshly transduced cells at day- 6 was collected and gene expression profiles of short-term expression of Pdx1 or Pdx1-VP16 were compared by RT-PCR.

As shown in Figure 3, short-term expression of Pdx1 or Pdx1-VP16 at day 6 (post-lentiviral transduction) activated genes related to pancreatic betacell development, including $H N F-1, N g n 3$, NeuroD, Glut-2, MafA, glucagon, and glucagon-like peptide-1 receptor (GLP1-R). Neither Pdx1 nor Pdx1-VP16 activated endogenous rat $P d \times 1, P a x 4, N K x 6.1$, Pax6, Isl-1, GK, insulin I, pancreatic polypeptide $(P P)$ or somatostatin (SS) gene expression. Interestingly, the major difference is noted in the expression of $N K \times 2.2$ and insulin II genes. Pdx1-VP16-expressing cells activated both $N k x 2.2$ and insulin II genes, whereas Pdx1-expressing cells failed to have any noticeable effect on such gene expression. These results indicate that both transcription factor genes are able to initiate the liver-to-pancreas transdifferentiation at a short-term, but Pdx1-VP16 is much more efficient in inducing expression of $\mathrm{Nkx} 2.2$ and insulin II genes.

\section{Gene Expression Profiles of Long-Term Expression of Pdx1 and Pdx1-VP16}

To determine the effects of long-term expression of Pdx1 or Pdx1-VP16 and the effects of glucose concentrations on the liver-pancreas transdifferentiation, we used single-cell-derived Pdx1- or Pdx1VP16-expressing clones and cultured the cells under 


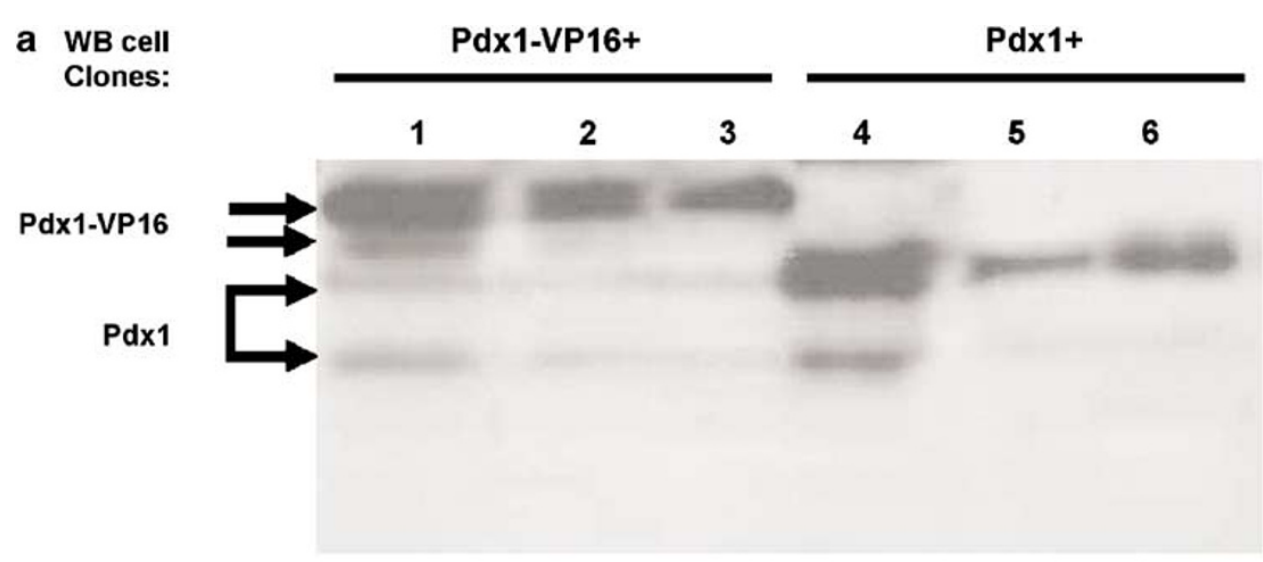

b

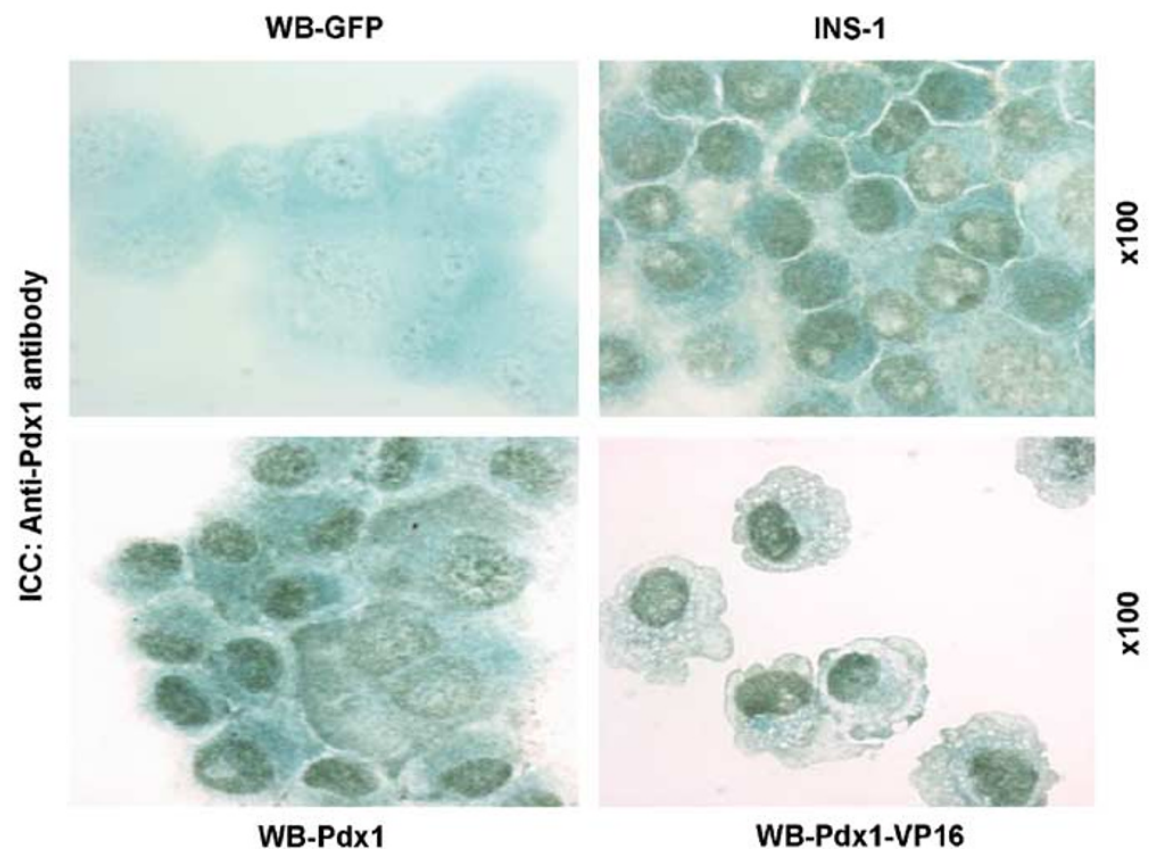

Figure 2 Confirmation of a persistent expression of Pdx1 or Pdx1-VP16 proteins and their nuclear localization. (a) Persistent expression of Pdx1 and Pdx1-VP16 proteins. Three positive clones from Pdx1 (clones 2, 4, and 9 in Figure 1a) or Pdx1-VP-expressing (1, 2 , and 7 in Figure 1b) clones were chosen to be continuously expanded for 3 months under normal culture condition (glucose 11.1 mM). The Pdx1or Pdx1-VP16 protein expression was examined as described in the methods and detected by anti-Pdx1 antibody. Lanes (1-3) represent Pdx1-VP16 expressing clones and lanes (4-6) Pdx1-expressing cell clones. Two arrows were placed in Pdx1 and Pdx1-VP16 proteins indicating phosphorylated (upper) or unphosphorylated (lower) forms, respectively. (b) Immunocytochemistry with anti-Pdx1 antibody. Cytospin slides were made from the 3-month expanded Pdx1- or Pdx1-VP16-expressing WB cells, WB-GFP cells (negative control), or rat insulinoma INS-1 cells (positive control). The slides were incubated with anti-Pdx1 antibody (1:4000) or rabbit serum (1:4000) (not shown) for $1 \mathrm{~h}$ at room temperature and secondary antibody (1:10000) for $30 \mathrm{~min}$. The pictures were taken under $\times 100$ oil lens.

either low-glucose $(5 \mathrm{mM})$ or high-glucose $(25 \mathrm{mM})$ conditions for different times (1,2, and 3 months). The same amount of total RNA collected at various times was reverse-transcribed, and the cDNA was used to amplify the genes related to beta-cell development and function. Figure 4 shows the comparisons of gene expression profiles following long-term expression of Pdx1- and Pdx1-VP16 protein under low- or high-glucose culture conditions. Rat insulinoma INS-1 (823/13) cells were used as positive controls (lane 1) and RNA samples without RT were performed to exclude DNA contamination (lane 2). As demonstrated in Figure 4, long-term persistent expression of Pdx1 or Pdx1-
VP16 under the low-glucose condition led to expression of endogenous $r P d x 1$, ngn3, NeuroD, Nkx2.2, isl-1, glucagons, MafA, and GK in both types of cells. Late-stage genes related to beta-cell development (Pdx4, Pax6, isl-1) and beta-cell function (insulin-I and II) were not detected under this low-glucose culture. Interestingly, in high-glucose culture, expression of many genes (Ngn3, NeuroD, Insulin II, HNF-1, Kir6.2, GK, and $P P$ ) was upregulated, with expression correlating with duration of culture under high-glucose conditions. These results demonstrated no major significant differences in the expression levels of the beta-cell related genes at both low and high-glucose culture conditions for 
88

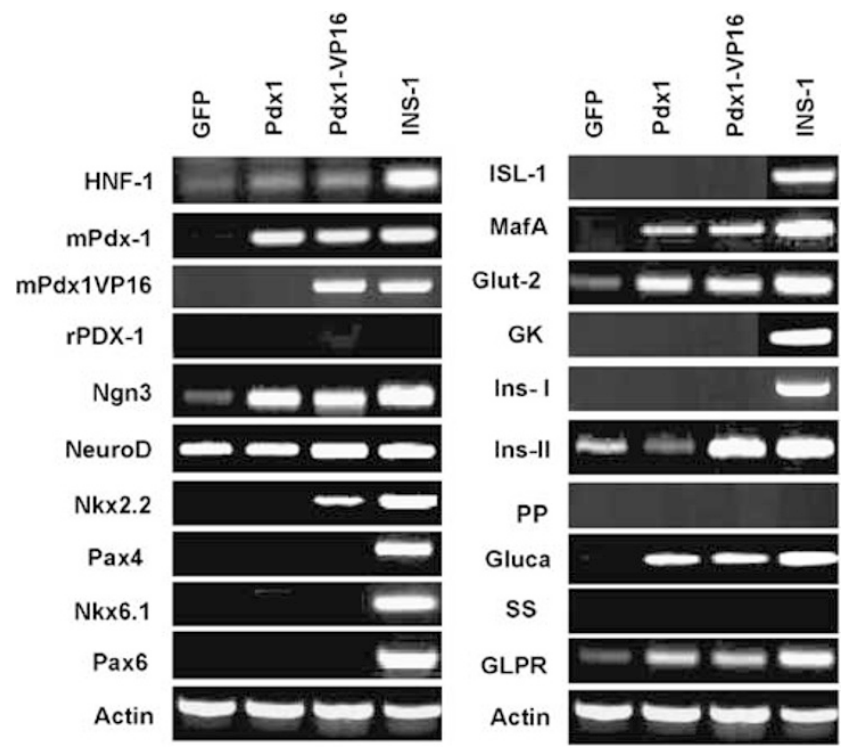

Figure 3 Profiles of short-term gene expression of Pdx1 or Pdx1VP16. Total RNA was extracted by trizol reagent and treated with DNAse. A total RNA ( $2 \mu \mathrm{g}$ ) was reversely transcribed and PCR was performed at 35 cycles and 1/5 of PCR products were analyzed by $1.8 \%$ of agarose gel and visualized with ethidium bromine. All primers were designed to cross intron (s) except for MafA and ngn3 (genes have only one exon) and the primer sequences are provided in Table 1. Rat insulinoma INS-1 (823/13) cells serve as positive controls for beta-cell gene expression. Parental WB cells transduced with LV-GFP serve as baseline for lentiviral vector control. Total RNA samples without RT were performed to exclude DNA contamination. Actin serves as house-keeping gene control to give relative expression levels in each sample. Pdx1 and Pdx1-VP16 plasmid were used as positive controls.

either Pdx1- or Pdx1-VP16-expressing cells. Both types of cells are able to respond to a prolonged high-glucose environment by differentiating and maturing along the pathway of beta-cell development.

Additional evidence for further cell differentiation along the beta-cell pathway is the pattern of hormonal gene expression. Upon short-term expression of either Pdx1 or Pdx1-VP16, both cell treatment groups expressed glucagon and insulin II genes, findings consistent with an early pancreatic precursor phenotype. ${ }^{18}$ However, glucagon gene expression became silent in the cells with long-term expression of Pdx1 or Pdx1-VP16, indicating more differentiated beta-like precursor cells. Therefore, we conclude that prolonged and persistent expres- sion of Pdx1 or Pdx1-VP16 reprograms the hepatic WB cells along the pancreatic beta-cell pathway to transdifferentiate into IPCs. However, short-term or transient expression of these transgenes resulted in a significant difference in terms of the efficiency of initiating liver-to-pancreas transdifferentiation. We also examined the expression profile of the liverrelated genes including albumin, cytokeratin 19 (CK19), hexokinase, and transforming growth factor $\alpha(T G F-\alpha)$ in the rat liver, parental WB cells, Pdx1and Pdx1-VP16-expressing cells. Figure 4c shows that gene expression of hexokinase and $T G F-\alpha$ does not change significantly after 3-month high-glucose culture. Albumin is not expressed in either native WB cells or those transduced with Pdx1 or Pdx1VP16. However, there is a noticeable reduction of CK19 gene expression in Pdx1-VP16-expressing cells, as compared to the level of CK19 in WB cells and Pdx1-expressing cells.

\section{Functional Studies of Liver-Derived Pancreatic Beta-Like Cells}

Our studies show that persistent expression of Pdx1 or Pdx1-VP16 in WB cells produces similar geneactivation profiles in these two sets of cells. Both sets of cells expressed lower levels of insulin II gene under low-glucose culture conditions, and higher levels of insulin II gene under high-glucose culture conditions. To determine whether these cells produce and release insulin, we measured cellular insulin content and insulin levels released into the culture medium in cells cultured under the maintenance condition (11.1 mM glucose). We found low levels of insulin content $(0.37 \pm 0.13 \mathrm{vs}$ $0.36 \pm 0.16 \mathrm{ng} / \mathrm{mg}$ protein) for Pdx1 and Pdx1-VP16expressing cells, respectively. There also was a low level of constitutive insulin release in Pdx1- and Pdx1-VP16-expressing cells after derivation of single-cell clones (data not shown), but the cells were not consistently sensitive to glucose stimulation. There was no significant difference between Pdx1VP16-expressing cells vs Pdx1-expressing cells in terms of insulin production and release.

We next examined the effectiveness of Pdx1- and Pdx1-VP16-expressing hepatic stem-like WB cells at reversing hyperglycemia in diabetic mice. Stzinduced diabetic NOD-scid mice $(n=$ three per group) were transplanted with Pdx1-, Pdx1-VP16,

Figure 4 Gene expression profiles of long-term expression of Pdx1- or Pdx1-VP16 under low or high culture conditions. (a) Expression of genes related to beta-cell development. (b) Expression of genes related to beta-cell function. Total RNA was extracted by triazol reagent and treated with DNAse; and $2 \mu \mathrm{g}$ of total RNA was reversely transcribed. All primers were designed to cross intron (s) except for mafA and ngn 3 , and the sequences are provided in Table 1. PCR was performed at 35 cycles and 1/5 of PCR products were analyzed by $1.8 \%$ of agarose gel and visualized with ethidium bromine. Rat insulinoma INS-1 cells serve as positive controls for beta-cell gene expression. Total RNA samples without RT serve as negative controls for RT-PCR products. Actin serves as house-keeping gene control to give relative expression levels in each sample. Samples were from Pdx1-expresssing cells cultured in LG and HG for 1, 2, and 3 months (left); and samples from Pdx1-VP16-expressing cells in LG and HG culture conditions for 1, 2, and 3 months are at right for both (a) and (b). (c) Expression of liver-related genes in WB-derived cells. Total RNA was extracted from parental WB cells, as well as Pdx1- and Pdx1-VP16 expressing WB cells after 3 months of high-glucose culture. Expression of the liver-related genes such as albumin, CK19, hexokinase, TGF- $\alpha$, was determined by RT-PCR. 
or GFP-expressing WB cells ( $\sim 2 \times 10^{6}$ cells/mouse) into the subrenal capsular space and the blood glucose levels were monitored every 3 days to observe the effect of these cells on the blood glucose levels. As demonstrated in Figure 5a, both Pdx1and Pdx1-VP16-expressing cells were capable

a

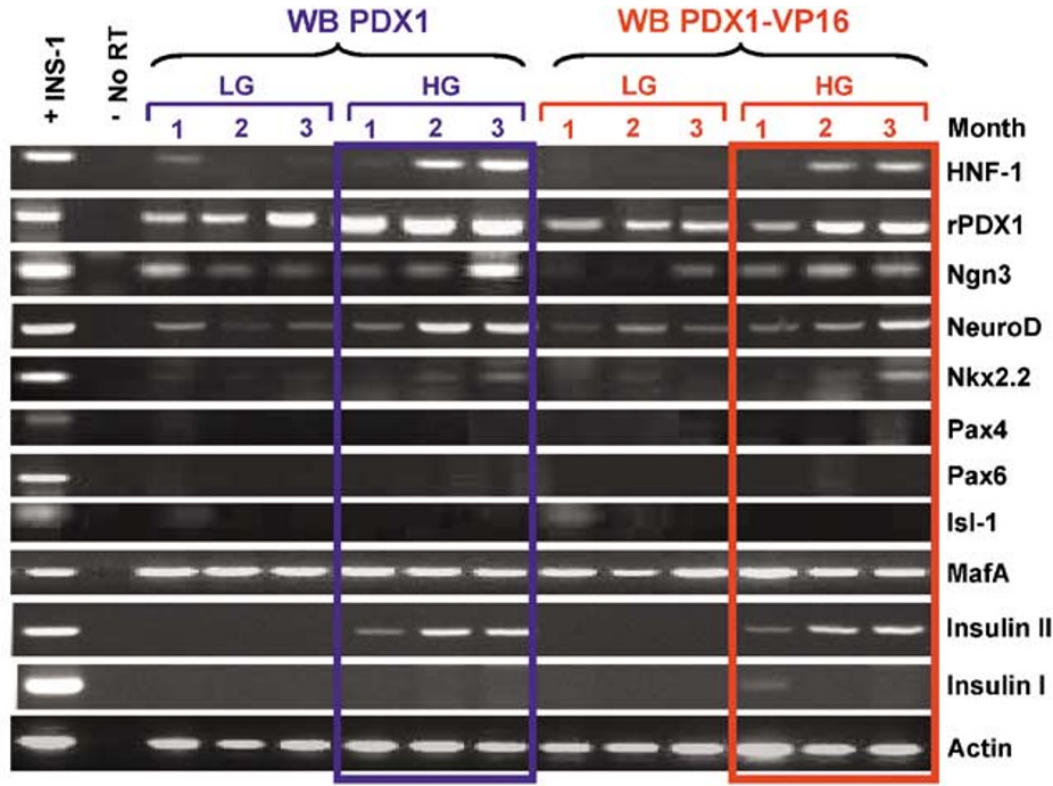

b
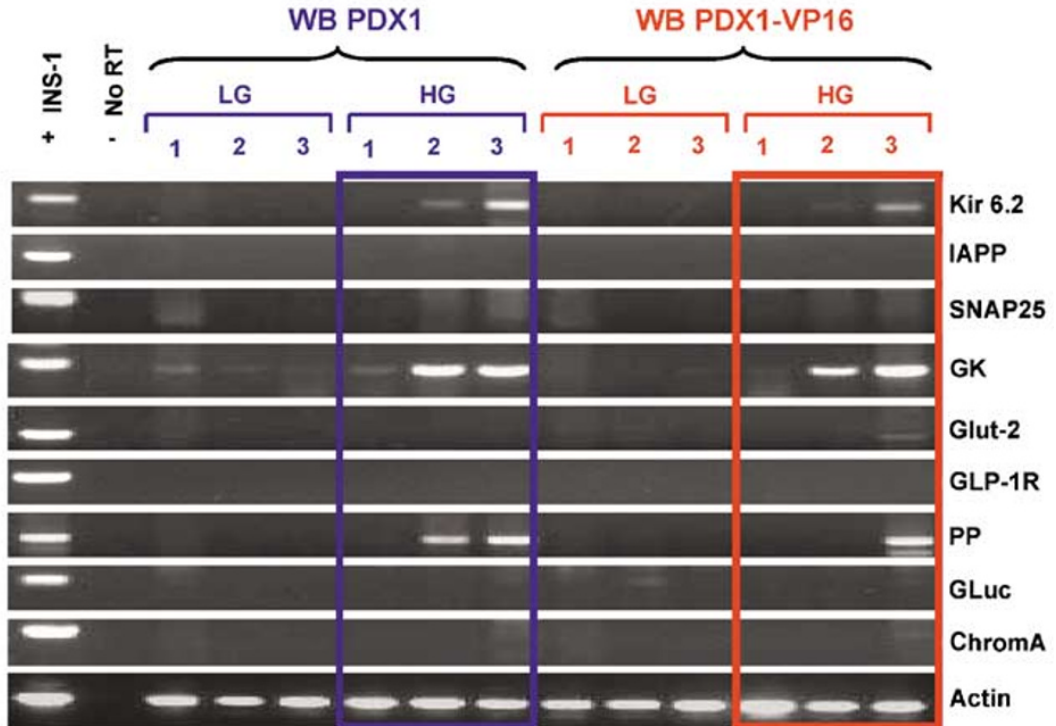

c

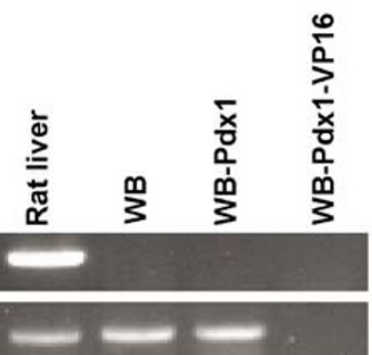

Hexokinase

TGF $a$

Actin 
90
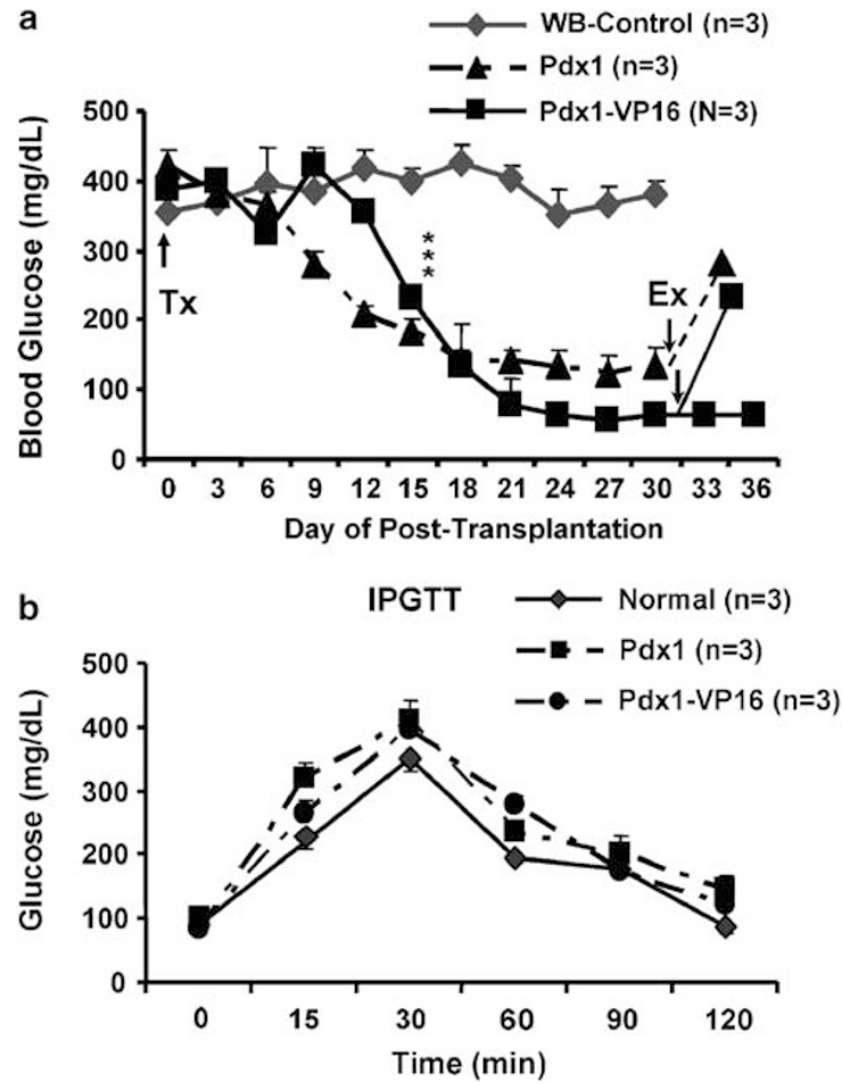

Figure 5 In vivo animal studies. (a) Reversal of hyperglycemia by transplanted Pdx1- or Pdx1-VP16-expressing WB cells. Singlecell-derived Pdx1-, Pdx1-VP16-expressing WB cells or WB-GFPexpressing control cells $\left(2 \times 10^{6}\right.$ cells/mouse) were transplanted (upward arrow, Tx) into Stz-induced diabetic mice after blood glucose reached around $400 \mathrm{mg} / \mathrm{dl}$ ( $n=3$ mice per group). The blood glucose levels were monitored by tapping the tail vein every three days. Left nephrectomy was performed on one mouse from each group (downward arrows, indicated by Ex), and blood glucose levels were measured $24 \mathrm{~h}$ after removal of left kidney. The animals were sacrificed at indicated times. Mean \pm s.e.m., $* * * P<0.001$. (b) Intraperitoneal glucose tolerance test (IPGTT). Mice received a loading dose of glucose ( $2 \mathrm{mg} / \mathrm{g}$ body weight) and the blood glucose levels were monitored at indicated times. The results represent the means \pm s.e.m. of the blood glucose levels of three mice in each group. Naïve mice were used as control mice for IPGTT. Mean \pm s.e.m., $n=3$ mice per group.

reversal of hyperglycemia within 3 weeks after transplantation. In contrast, the mice receiving GFP-expressing WB cells showed no reduction in their blood glucose levels and maintained a persistent hyperglycemia. Removal of the transplanted cells by left nephrectomy in one mouse from each group resulted in rebound hyperglycemia, indicating that amelioration of hyperglycemic effects is the direct consequence of the transplanted Pdx1- or Pdx1-VP16-expressing cells. To further determine the ability of the transplanted, genetically modified, liver-derived IPCs to respond to a glucose challenge, the mice were given a loading dose of glucose, and the blood glucose levels were monitored as described in the methods. As shown in Figure 5b,
IPGTT showed that those mice receiving the transplanted Pdx1- or Pdx1-VP16-expressing cells have a similar pattern to that observed with normal control mice without cell transplantation. This finding suggests that these liver-derived IPCs indeed further differentiate and mature into functional pancreatic beta-like cells. We may thus conclude that both Pdx1- and Pdx1-VP16-expressing WB cells are capable of differentiating and maturing into functional IPCs that reverse diabetes in mice.

\section{Discussion}

Forced expression of the 'master control gene' $\mathrm{Pdx}^{4,5}$ or its modified form Pdx1-VP16 ${ }^{9,19}$ reprograms liver cells to take different, but related, differentiation pathways toward pancreatic fate. Controversy arose as to whether expression of Pdx1 alone is sufficient to convert the liver cells into pancreatic beta cells. ${ }^{4,6}$ Several studies showed that transient ectopic expression of Pdx1 or Pdx1VP16 mediated by adenovirus vectors successfully activated genes encoding pancreatic exocrine enzymes, ${ }^{4,6,19}$ but both resulted in unwanted production and release of pancreatic exocrine enzymes to the liver, causing harmful hepatitis in the liver parenchyma. ${ }^{6,7}$ More importantly, transient expression of only Pdx1 in mouse liver failed to convert sufficient numbers of hepatocytes into IPCs, whereas the combined expression of transcription factor NeuroD and beta-cell promoting factor betacellulin in the mouse liver successfully converted the liver cells into sheets of precursor of pancreatic endocrine cells. ${ }^{6}$ That study raised the possibility that additional transcription factors or further modification of Pdx1 may be required to reliably transduce liver cells along the pancreatic endocrine differentiation pathway. We previously demonstrated that persistent expression of Pdx1-VP16 fusion protein, as mediated by LV, selectively transdifferentiated hepatic WB stem-like cells into functional IPCs without evidence of pancreatic exocrine differentiation. ${ }^{9}$ These liver-derived IPCs completely normalized hyperglycemia in the Stzinduced diabetic NOD-scid mice. Recent studies from Japanese groups compared the in vivo effect of adenovirus-mediated transient expression of $\mathrm{Pdx} 1$ and Pdx1-VP16 on glucose levels of diabetic mice and concluded that Pdx1-VP16 is superior to Pdx1 expression in the efficiency of conversion of the liver cells into IPCs and reduction of hyperglycemia in mice. ${ }^{20,21}$ However, studies from Ferber's group have shown that expression of $\mathrm{Pdx} 1$ alone is sufficient to convert the mouse liver cells into sufficient number of IPCs which have a lasting effect on reversal of experimental diabetes in mice. ${ }^{5}$

In order to resolve these unsettled issues, we tested the hypothesis that vector efficiency may be an obstacle in characterizing Pdx1-mediated reprogramming of liver cell differentiation into pancreatic 
beta cells. In the current study, we used LV for systematic comparison of the effects of both shortterm and long-term expression of $\mathrm{Pdx} 1$ or Pdx1VP16 in hepatic stem-like WB cells on gene expression profiles as well as beta-cell functional studies. In short-term experiments, we transduced WB cells with LV-Pdx1 or LV-Pdx1-VP16 and obtained total RNA from the resulting cells (day-6 post-transduction) for evaluation of the effects of short-term expression profiles of the transgenes. LV-Pdx1-VP16 was found to be more effective than LV-Pdx1 in initiating liver-to-pancreas transdifferentiation. Both Pdx1- and Pdx1-VP16-expressing cells activated insulin II and glucagon genes (Figure 3), a pattern of coexpression consistent with reported features of early pancreatic endocrine precursor cells. ${ }^{18}$ These precursor cells have potential to further differentiate into functional IPCs in a conducive microenvironment, as evidenced by our next experiments.

For long-term studies, we transduced WB hepatic stem-like cells with mPdx1- and mPdx1-VP16 LV and generated single-cell-derived Pdx1 or Pdx1VP16-expressing clones for the comparison studies in gene expression profiles and in functional studies carried out in vitro and in vivo. Because $\mathrm{Pdx} 1$ plays an essential role in pancreas development and in maintaining beta-cell function, ${ }^{1}$ our long-term Pdx1 and Pdx1-VP16 expression studies were performed, under either low- or high-glucose culture conditions. Our results indicated that persistent long-term expression of either Pdx1 or Pdx1-VP16 proteins resulted in similar expression profiles of genes including pancreatic transcription factors related to beta-cell development (HNF1, rPdx1, Ngn3, NeuroD, Nkx2.2, Nkx6.1, Pax4, Pax6, Isl-1, MafA) and pancreatic endocrine hormones (glucagon, $P P$, insulin $I$ and insulin $I I$ ), and genes related to the regulation of beta-cell function (Kir6.2, snare 25, IAPP, GK, Glut-2, chromagranin A, and GLP-1R). ${ }^{22,23}$ Specifically, persistent expression of Pdx1 or Pdx1VP16 in the hepatic WB cells activated upstream genes (rPdx1, Ngn3, NeuroD, and Nkx2.2) as observed in early beta-cell development; however, they failed to activate late-stage genes (Pax4, Isl-1, and $P a x 6$ ), as well as other functional genes (GK, Glut-2, Kir6.2, snare 25). We previously demonstrated that IPC can be obtained either by long-term high-glucose culture ${ }^{17}$ or by expressing Pdx1-VP 16 in liver stem cells. ${ }^{9}$ Indeed, when the cells were previously cultured in a high-glucose medium for prolonged periods, many genes (ngn3, neuroD, Nkx2.2, insulin II, GK, Kir6.2, and PP) were gradually upregulated and reached peak expression after 60-90 days. In the current study, we observed that glucagon expression ceased during the highglucose culture of either Pdx1- or Pdx1-VP16 expressing cells.

These patterns in gene expression profiles are consistent with progressive pancreatic beta-cell differentiation and maturation, moving from short- term to long-term expression of Pdx1- or Pdx1-VP16. However, some late-stage transcription factors such as Pax4, isl-1, and Pax6 were not expressed, suggesting that additional external factors ${ }^{24}$ or a diabetic microenvironment are needed for their activation. $^{9}$ As demonstrated in Figure 5, both Pdx1- and Pdx1-VP16-expressing WB cells were able to reverse diabetes by normalizing blood glucose levels within 3 weeks after transplantation into the diabetic mice, and they both show a similar pattern in response to a glucose challenge, as indicated in IPGTT. Such findings demonstrate that liver-derived IPCs can become fully functional when exposed to the in vivo diabetic microenvironment. In the latter instance, there is no dramatic difference between the effects of long-term expression of Pdx1 and Pdx1-VP16 in terms of gene expression in vitro, and capacity of becoming functional IPCs in the diabetic microenvironment in vivo. The powerful effects of the diabetic microenvironment in vivo may be due to both hormonal factors, such as hyperglycemia and cell-cell interactions.

In our ongoing work, we have systematically compared the implanted cells to native pancreatic beta cells with respect to their histology, immunostaining (for insulin, Pdx1, glucagon, Islet 1, and Nkx6.1 proteins), and their ultrastructural characteristics by electron microscopy. Both Pdx1- and Pdx1-VP16-expressing implanted cells express strong insulin protein as well as pancreatic beta cell-specific transcription factors $\mathrm{Pdx} 1$, islet-1, and Nkx6.1, but not glucagon, and these results will be presented in a separate report.

Most published in vivo studies delivered the Pdx1 gene to the liver cells by adenoviral gene transfer, 4,6,20 inducing transient expression of Pdx1, which typically peaked within a week, then fell off rapidly and disappeared after 2 weeks. ${ }^{20,25}$ For this reason, we deliberately chose to examine the short-term gene expression profiles between Pdx1and Pdx1-VP16-expressing WB cells at day-6 after lentiviral transduction. Our results indicated that expression of Pdx1-VP16 short-term is more efficient than Pdx1 in initiating the process of the liver-to-endocrine transdifferentiation (eg NKx2.2 activation) and inducing insulin II gene expression (Figure 3). Although fetal neuronal cells may share a similar developmental pathway at the early developmental stage as to the early stage of pancreatic beta cells by expressing Ngn3, insulin II, and Nkx2.2, our complete gene expression profile as well as our in vivo animal studies indicate that forced expression of Pdx1- and Pdx1-VP16 in WB cells indeed redirected the liver stem cells towards the developmental pathway for pancreatic beta cells. Since Pdx1 has been shown to activate its target genes by association with several cofactors such as $\mathrm{PBX}^{26,27}$ and the expression of these protein partners are absent in the liver, a possible explanation for the effectiveness of Pdx1-VP16 expression in initiating transdifferentiation and generating earlier IPCs in 
the liver is that the powerful activation domain of VP16 potentiates transcription of the gene to which it is fused ${ }^{8,28}$ by recruiting the target genes to the proximity of $\mathrm{Pdx} 1$ without the need for other cofactor proteins. ${ }^{9,19}$ Interestingly, neither Pdx1 nor Pdx1-VP16 expression at day 6 could induce endogenous rat $\mathrm{Pdx} 1$ activation (Figure 3), suggesting a longer time and sustained dosage of Pdx1 or Pdx1-VP16 expression is needed for the activation of endogenous $\mathrm{Pdx} 1$. It is possible that the variations of the timing and dosage for Pdx1 or Pdx1-VP16 expression in an individual liver cell are partially responsible for the various effects of adenovirusmediated Pdx1 expression in the mouse liver on the degree of the reducing hyperglycemia in diabetic mice and on the numbers of IPCs identified in the liver observed by different investigators. ${ }^{4,6}$

In summary, short-term $P d x 1-V P 16$ expression in hepatic stem-like WB cells is more efficient than $P d \times 1$ expression in its ability to initiate liver-topancreas transdifferentiation. However, long-term, persistent expression of either Pdx1 or Pdx1-VP16, as mediated by LV, results in similar profiles of gene expression. Both Pdx1 and Pdx1-VP16 promote pancreatic beta-like cell differentiation upon longterm high-glucose induction in vitro and after transplanting these transduced cells into diabetic mice. To the best of our knowledge, this is the first study to use a highly efficient and persistent expression vector to systematically probe Pdx1- or Pdx1-VP16-mediated liver-to-pancreas transdifferentiation at multiple levels of molecular, cellular, and whole animals. The information presented here demonstrates the great promise of lentivectors and provides new clues on the reprogramming of liver cells to generate beta-cell surrogates for cell replacement therapy in treating and/or curing diabetes.

\section{Acknowledgements}

We thank Christopher V Wright for providing Pdx1 cDNA and Pdx1 antibody, Christopher B Newgard for rat INS-1 cell line (clone 832/13), WB Coleman for WB cell line and Marko E Horb for Pdx1-VP16 plasmid. National Institutes of Health research grants (DK064054, DK068031, DK071186, and DK071831 to L-J Yang) supported this research project.

\section{References}

1 Habener JF. The role of pancreatic duodenum homeobox protein-1 in the development of diabetes mellitus. Drug News Perspect 2002;15:491-497.

2 Stoffers DA, Zinkin NT, Stanojevic V, et al. Pancreatic agenesis attributable to a single nucleotide deletion in the human IPF1 gene coding sequence. Nat Genet 1997;15:106-110.
3 Hui H, Perfetti R. Pancreas duodenum homeobox-1 regulates pancreas development during embryogenesis and islet cell function in adulthood. Eur J Endocrinol 2002;146:129-141.

4 Ferber S, Halkin A, Cohen $\mathrm{H}$, et al. Pancreatic and duodenal homeobox gene 1 induces expression of insulin genes in liver and ameliorates streptozotocininduced hyperglycemia. Nat Med 2000;6:568-572.

5 Ber I, Shternhall K, Perl S, et al. Functional, persistent, and extended liver to pancreas transdifferentiation. J Biol Chem 2003;278:31950-31957.

6 Kojima H, Fujimiya M, Matsumura K, et al. NeuroDbetacellulin gene therapy induces islet neogenesis in the liver and reverses diabetes in mice. Nat Med 2003;9:596-603.

7 Miyatsuka T, Kaneto H, Kajimoto Y, et al. Ectopically expressed PDX-1 in liver initiates endocrine and exocrine pancreas differentiation but causes dysmorphogenesis. Biochem Biophys Res Commun 2003;310: 1017-1025.

8 Sadowski I, Ma J, Triezenberg S, et al. GAL4-VP16 is an unusually potent transcriptional activator. Nature 1988;335:563-564.

9 Cao LZ, Tang DQ, Horb ME, et al. High glucose is necessary for complete maturation of pdx-1-vp16expressing hepatic cells into functional insulin-producing cells. Diabetes 2004;53:3168-3178.

10 Tang DQ, Cao LZ, Burkhardt BR, et al. In vivo and in vitro characterization of insulin-producing cells obtained from murine bone marrow. Diabetes 2004;53: 1721-1732.

11 Chang LJ, Zaiss AK. Lentiviral vectors. Preparation and use. Methods Mol Med 2002;69:303-318.

12 Chang LJ, Zaiss AK. Self-inactivating lentiviral vectors and a sensitive Cre-loxP reporter system. Methods Mol Med 2003;76:367-382.

13 Chang LJ, Urlacher V, Iwakuma $\mathrm{T}$, et al. Efficacy and safety analyses of a recombinant human immunodeficiency virus type 1 derived vector system. Gene Therapy 1999;6:715-728.

14 Grisham JW, Coleman WB, Smith GJ. Isolation, culture, and transplantation of rat hepatocytic precursor (stemlike) cells. Proc Soc Exp Biol Med 1993;204:270-279.

15 Couchie $\mathrm{D}$, Holic $\mathrm{N}$, Chobert $\mathrm{MN}$, et al. In vitro differentiation of WB-F344 rat liver epithelial cells into the biliary lineage. Differentiation 2002;69: 209-215.

16 Hohmeier HE, Mulder H, Chen G, et al. Isolation of INS-1-derived cell lines with robust ATP-sensitive $\mathrm{K}+$ channel-dependent and -independent glucose-stimulated insulin secretion. Diabetes 2000;49:424-430.

17 Yang $\mathrm{L}$, Li S, Hatch $\mathrm{H}$, et al. In vitro trans-differentiation of adult hepatic stem cells into pancreatic endocrine hormone-producing cells. Proc Natl Acad Sci USA 2002;99:8078-8083.

18 Teitelman G, Alpert S, Polak JM, et al. Precursor cells of mouse endocrine pancreas coexpress insulin, glucagon and the neuronal proteins tyrosine hydroxylase and neuropeptide Y, but not pancreatic polypeptide. Development 1993;118:1031-1039.

19 Horb ME, Shen CN, Tosh D, et al. Experimental conversion of liver to pancreas. Curr Biol 2003;13: 105-115.

20 Imai J, Katagiri $\mathrm{H}$, Yamada $\mathrm{T}$, et al. Constitutively active PDX1 induced efficient insulin production in adult murine liver. Biochem Biophys Res Commun 2005;326:402-409. 
21 Kaneto H, Nakatani Y, Miyatsuka T, et al. PDX-1/VP16 fusion protein, together with NeuroD or Ngn3, markedly induces insulin gene transcription and ameliorates glucose tolerance. Diabetes 2005;54:10091022.

22 Wilson ME, Scheel D, German MS. Gene expression cascades in pancreatic development. Mech Dev 2003; 120:65-80.

23 Blakolmer K, Jaskiewicz K, Dunsford HA, et al. Hematopoietic stem cell markers are expressed by ductal plate and bile duct cells in developing human liver. Hepatology 1995;21:1510-1516.

24 Ramiya VK, Maraist M, Arfors KE, et al. Reversal of insulin-dependent diabetes using islets generated in vitro from pancreatic stem cells. Nat Med 2000;6: 278-282.
25 Peeters MJ, Patijn GA, Lieber A, et al. Adenovirusmediated hepatic gene transfer in mice: comparison of intravascular and biliary administration. Hum Gene Ther 1996;7:1693-1699.

26 Dutta S, Gannon M, Peers B, et al. PDX:PBX complexes are required for normal proliferation of pancreatic cells during development. Proc Natl Acad Sci USA 2001; 98:1065-1070.

27 Peshavaria M, Cissell MA, Henderson E, et al. The PDX-1 activation domain provides specific functions necessary for transcriptional stimulation in pancreatic beta-cells. Mol Endocrinol 2000;14:1907-1917.

28 Triezenberg SJ, Kingsbury RC, McKnight SL. Functional dissection of VP16, the trans-activator of herpes simplex virus immediate early gene expression. Genes Dev 1988;2:718-729. 